\title{
Probe-Corrected Near-Field to Far-Field Transformation Using Multiple Spherical Wave Expansions
}

\author{
Fernando Rodríguez Varela, Belén Galocha Iragüen, Manuel Sierra Castañer \\ Radiation Group, Information Processing Telecommunications Centre, ETSI Telecomunicación \\ Universidad Politécnica de Madrid \\ Av. Complutense $n^{\circ}$ 30, Madrid, Spain \\ \{f.rodriguezv, belen.galocha, manuel.sierra \}@upm.es
}

\begin{abstract}
Near-field to far-field transformations constitute a powerful antenna characterization technique for near-field measurement scenarios. In this paper a near-field to far-field transformation technique based on multiple spherical wave expansions is presented. Thanks to its iterative matrix inversion nature, the approach performs the transformation of fields measured on arbitrary surfaces. Also, irregular sampling schemes can be incorporated. The proposed algorithm is based on modelling the antenna fields with not one, but several spherical wave expansions distributed over its geometry. Due to the high number of spherical wave expansions, their truncation number can be arbitrarily reduced. Working with expansions of low order allows to incorporate the probe correction in the transformation in a very simple way, accepting any type of probe and orientation. Only the probe far-field pattern is used, thus working with its full spherical wave expansion is avoided. The algorithm is validated using simulated field data as well as measurements of real antennas.
\end{abstract}

Index Terms-near-field to far-field transformation, spherical wave expansion, near-field measurements, probe correction.

\section{INTRODUCTION}

Antenna near-field measurements constitute a versatile tool for determining antennas radiation pattern. This type of measurements requires the use of post processing techniques to transform the antenna under test (AUT) measured nearfields into the radiated far-field. Traditionally, near-field measurements have been performed using canonical acquisition surfaces to simplify the post processing steps: spheres, cylinders and planes. From the three, the spherical measurement system has been regarded as one of the most accurate and powerful techniques [1]. With the growing interest in high frequencies and robotic positioning equipment [2], achieving correct probe location and orientation becomes challenging. In addition, there exists an increasing trend in measurement set-ups where the antenna near-field is measured in a surface of arbitrary shape and sampling enclosing the AUT, as in the case of over-the-air (OTA) measurements [3]. Near-field to far-field transformation algorithms suitable for these scenarios must be implemented, as the classical post processing techniques cannot be applied in these cases.
Most field transformation techniques working on arbitrary surfaces consist in a two-step procedure: First, an equivalent representation of the AUT is found by solving an inverse problem with the knowledge of the near-field. Then, the farfield of the equivalent representation is computed to obtain the AUT radiation pattern. Depending on the type of equivalent representation used (magnetic/electric currents [4], spherical waves [5], complex source beams [6]) different approaches can be followed to formulate the problem and reduce the number of operations. This is an essential aspect as the solution of these type of inverse problems shows a scalability of $O\left(S^{4}\right)$, being $S$, the antenna electrical size. The bad scalability can pose performance issues for electrically big antennas in terms of memory and computation time.

In this paper, a multiple spherical wave expansion representation is used to model the AUT. This is done defining a set of unknown spherical wave expansions centered around the antenna shape, as opposed to the conventional approach based on a single spherical wave expansion centered at the coordinate system origin. The advantage of using several expansions is that the probe-correction can be applied using directly the probe's far-field pattern without having to deal with its own spherical wave expansion and complex translation and rotation coefficients. In addition, the approach is suitable for a multi-level extension, which could reduce the algorithm computational complexity drastically.

The structure of this paper is as follows. In Section II, the basic theory of the multiple spherical wave expansion field transformation algorithm is presented. In section III, the approach is validated based on simulations of antenna fields, leaving the verification with real measured data for section IV. Section V concludes this paper.

\section{NEAR-FIELD TO FAR-FIELD TRANSFORMATION}

The field $\vec{E}$ radiated by a given antenna admits a spherical wave expansion (SWE) [7] that can be centered at an arbitrary point $\vec{r}_{i}$ :

$$
\vec{E}(\vec{r})=\sum_{s=1}^{2} \sum_{n=1}^{N} \sum_{m=-n}^{n} Q_{s m n} \vec{F}_{s m n}^{(3)}\left(\vec{r}-\vec{r}_{i}\right)
$$


where $\vec{F}_{s m n}^{(3)}(\vec{r})$ are the spherical vector wave functions; $Q_{s m n}$, the antenna spherical coefficients, and $N$ the truncation number of the expansion. This truncation number is related to the degrees of freedom needed to model the antenna field variations and it is proportional to its size. The bigger the antenna is, the more variations will experiment the radiated field and thus more spherical harmonics are needed to model it.

Conventionally $\vec{r}_{i}$ is set to 0 and the coordinate system is centered at the antenna system to minimize $N$ and so, the number of terms in the summation. In this case, a different approach will be taken, as the field $\vec{E}(\vec{r})$ can be modeled also using not one, but several SWEs centered at different points $\vec{r}_{i}$ :

$$
\vec{E}(\vec{r})=\sum_{i=1}^{I} \sum_{s=1}^{2} \sum_{n=1}^{N_{i}} \sum_{m=-n}^{n} Q_{s m n}^{i} \vec{F}_{s m n}^{(3)}\left(\vec{r}-\vec{r}_{i}\right)
$$

The combination of several SWEs creates new field variations so each individual SWE needs now a lower truncation number $N_{i}$ than in the previous case. The lower the values of $N_{i}$ used, the smaller the number of harmonics will have each expansion, so more expansions are needed to model adequately the field variations. Fig. 1 shows a schematic representation of the modeling of a reflector antenna using six SWEs distributed over its surface.

In a real measurement scenario, it is not possible to measure directly the AUT field $\vec{E}(\vec{r})$ since the antenna used as probe has some influence. The probe has its own spherical wave expansion that can be introduced in the above formulation to consider its effect. The probe SWE coefficients must be rotated and translated depending on its location and orientation leading to complex calculations. In addition, if the probe exhibits non-ideal orientations, more rotations are needed [8]. However, if the truncation number of the expansions $N_{i}$ is set to a low value compared to the probe distance, the signal measured by the probe can be approximated by:

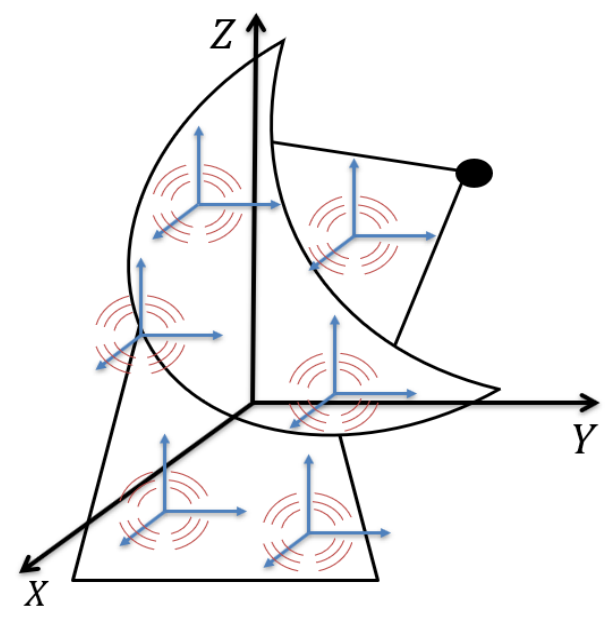

Fig. 1. Modelling of a reflector antenna with six SWEs distributed over its surface.

$$
\sum_{i=1}^{I} \vec{P}\left(\vec{r}_{i}-\vec{r}\right) \cdot \sum_{s=1}^{2} \sum_{n=1}^{w(\vec{r}) \approx} \sum_{m=-n}^{n} Q_{s m n}^{i} \vec{F}_{s m n}^{(3)}\left(\vec{r}-\vec{r}_{i}\right)
$$

Being $\vec{P}(\vec{r})$ the probe radiation pattern. Note that $\vec{r}_{i}-\vec{r}$ represents the angular direction of the $i$ th SWE seen from the probe placed at $\vec{r}$. Therefore, it can be considered that the measured signal $w(\vec{r})$ is a superposition of the contributions of each SWE weighted by the probe radiation pattern in the angular direction where the SWE is seen from the probe.

Eq. (3) can be expressed as a matrix-vector product:

$$
W=C Q
$$

being $W$ a vector that contains the probe measurements in all the acquisition points, $Q$ the vector that contains the coefficients of all SWEs and $C$ the coupling matrix that performs the summation and multiplications. Our problem is to determine $Q$ from the field measurements $W$, so we can evaluate the equivalent representation $Q$ at the far-field using the asymptotic form of (2) when $\vec{r} \rightarrow \infty$.

Eq. (4) is solved in the least squares sense by computing:

$$
Q=\left(C^{H} C\right)^{-1} C^{H} W
$$

Due to the high number of unknowns involved, an iterative matrix inversion method such as Conjugate Gradient (CG) [9] is preferred over a direct inversion. To reach a valid solution, well distributed and sampled measurements $W$ are needed for two orthogonal polarizations. There are no further restrictions, so the method is suitable for arbitrary measurement grids with irregular sampling.

\section{TRANSFORMATION RESULTS}

The capabilities of the proposed transformation algorithm are validated using a simulation example with analytical data. Considered is a distribution of 600 Hertzian dipoles randomly placed at plane $z=0$ and fed by voltages ranging between 0 and $1 \mathrm{~V}$. The distribution has an approximate size of $3 \lambda \times 3 \lambda$. The electric field radiated by the distribution is measured over a non-canonical surface given by the following parametric expression in spherical coordinates:

$$
r(\theta, \varphi)=8\left(1+(\cos \theta \sin \varphi)^{2}\right) \lambda
$$

with a sampling of 40 points in $\theta$ and 80 in $\varphi$. Fig. 2 depicts the dipole distribution (in black) and the acquisition surface cut in half for better visualization.

The probe antenna used is modelled with a radiation pattern following a $\cos ^{q} \theta$ pattern with $q=8$. The probe has been selected to be directive for better demonstration of the algorithm probe correction capabilities. The effect of the probe influence can be appreciated in Fig. 3 where a $\varphi=0^{\circ}$ field cut has been depicted, along with the signal measured by the probe. The effect is significant as the measurement surface is very close to the AUT. 


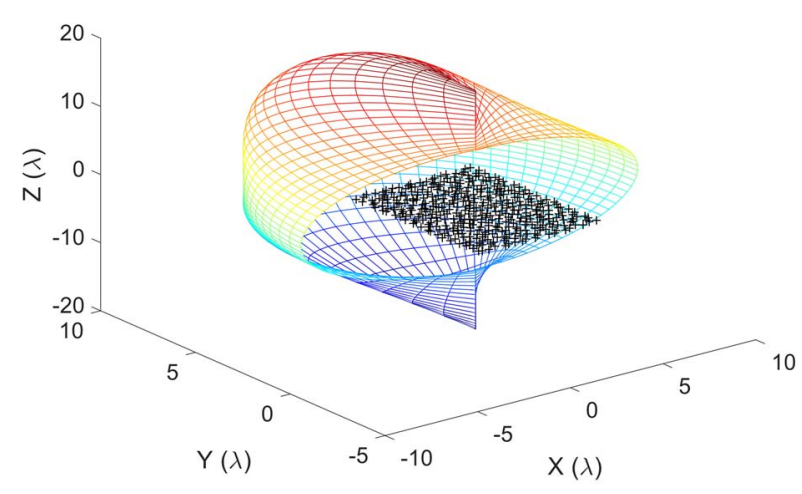

Fig. 2. Dipole distribution and measurement surface for the near- field

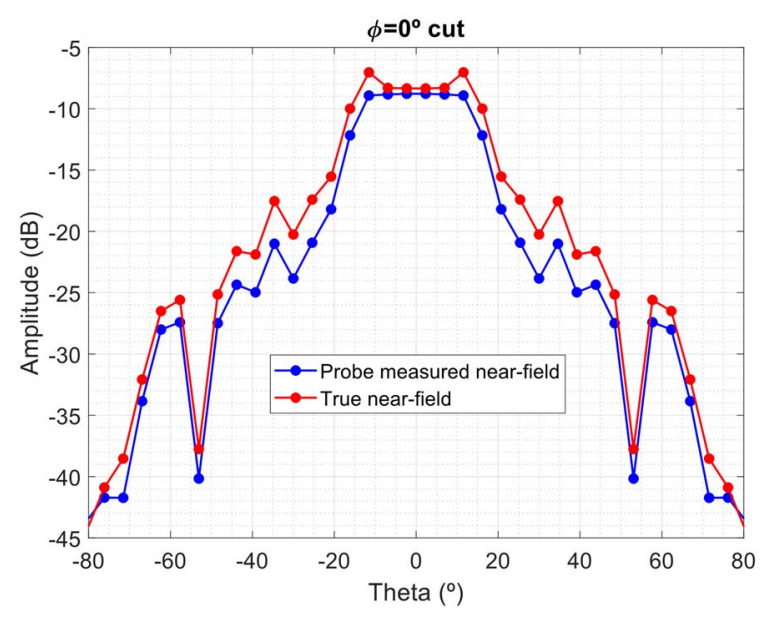

Fig. 3. Comparison of the radiated near-field and the measured field by a directive probe.The last is used as input for the transformation algorithm.

To perform the near-field to far-field transformation, the antenna is modelled as a set of SWEs centered at $z=0$ covering its surface. As mentioned in the previous section, the number of expansions needed depends on the truncation number $N_{i}$. Two cases of truncation number have been investigated for this example $N_{i}=2$ and $N_{i}=6$ for all expansions. In Fig. 4 the model for the two cases is depicted, where the centers of each of the SWE are signaled with red circles. It is observed that the case of $N_{i}=2$ requires smaller spacing between SWEs since the modal content of the expansions is lower than in the case of $N_{i}=6$. With this SWE distribution, matrix $C$ is built and vector $W$ is populated with the values measured by the probe.

The near-field to far-field transformation is performed for the two cases using the Conjugate Gradient algorithm to obtain the coefficients of each SWE. The transformed far-field is compared with the analytically computed field of the array distribution to obtain the transformation error. In Fig. 5 the errors for the two cases have been depicted along with the analytical field. In addition, the error of a non-probe-corrected transformation has been superposed. It can be seen that neglecting the probe effect yields an error with a level of roughly $-10 \mathrm{~dB}$ with respect to the reference field. Using
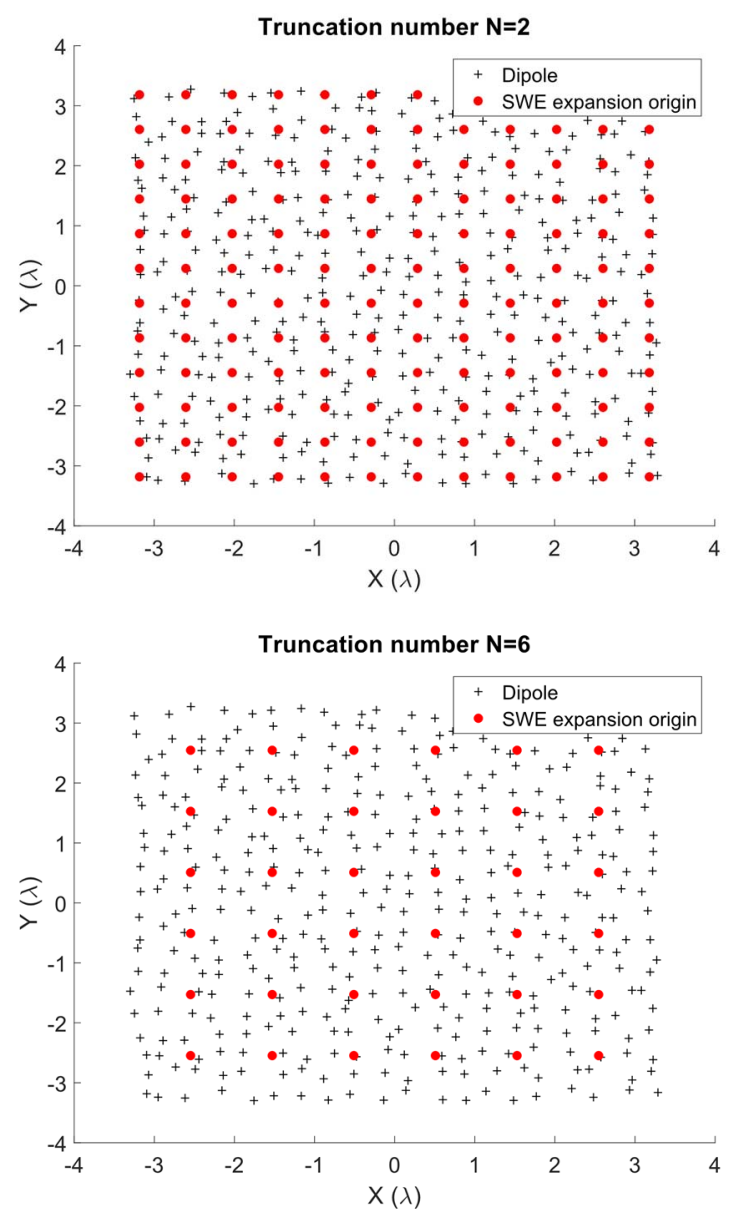

Fig. 4. Different configurations for the same dipole array antenna

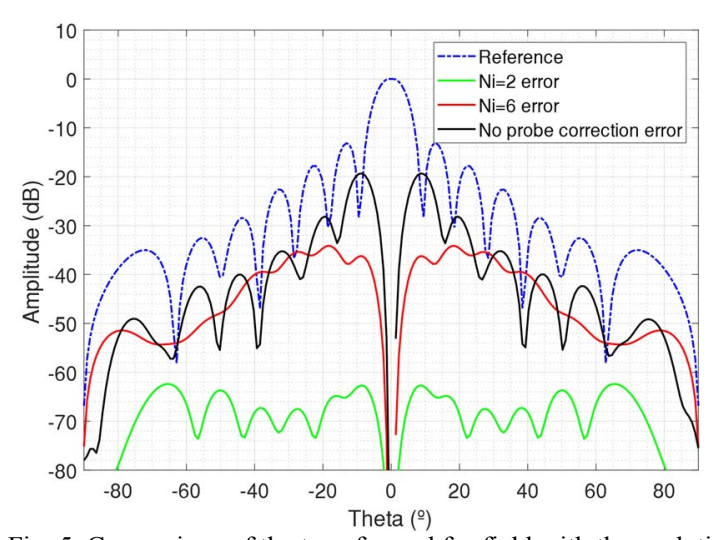

Fig. 5. Comparison of the transformed far-field with the analytical solution for several SWE configurations

multiple spherical wave expansions allows to include the probe effect reducing this error significantly.

However, there exists some residual error due to the approximation made in (3). This approximation is more ambitious for the case of $N_{i}=6$ and the error exhibited is still relevant. For less directive probes and/or larger measurement 
distances, higher values of $N_{i}$ could be used maintaining negligible transformation error levels. If the probe distance is large enough, a single SWE could give enough accuracy and the proposed algorithm would be identical to the approach proposed in [5].

\section{TRANSFORMATION OF NEAR-FIELD MEASURED DATA}

Finally, the proposed algorithm is verified using actual near-field data to check its stability against noise and practical inaccuracies. The test antenna will be a $60 \mathrm{~cm}$ diameter reflector operating at $8 \mathrm{GHz}$. Since there was not hardware available to generate a measurement surface different from a sphere, plane or cylinder or with irregular sampling, the selected shape has been a half sphere. This acquisition is generated performing a standard spherical near-field measurement truncating the rest of the sphere. Naturally, the truncated part corresponds to the backside of the antenna, so that most of the radiated field is kept. This corresponds to the region $\theta \leq 90^{\circ}$ (see Fig. 6 for the axes definition). The sampling rate is given by the standard spherical criterion [7]:

$$
\left\{\begin{array}{c}
\Delta \theta=\frac{\pi}{N+1} \\
\Delta \varphi=\frac{2 \pi}{2 N+1}
\end{array}\right.
$$

with $N=\left[\frac{2 \pi}{\lambda} r_{0}\right]+10$ and $r_{0}$ being the AUT minimum sphere radius. The brackets indicate the largest integer smaller than or equal to the number inside them.

Measurements for two orthogonal polarizations are performed at a distance of $5 \mathrm{~m}$. At this distance the probe effect is small, but the algorithm is taken to the limit using local SWEs of minimum order $N_{i}=1$. The approximate spacing between SWEs is set to around $0.35 \lambda$. From the measurements and problem geometry, vector $W$ and matrix $C$ are populated respectively and the Conjugate Gradient algorithm is started.

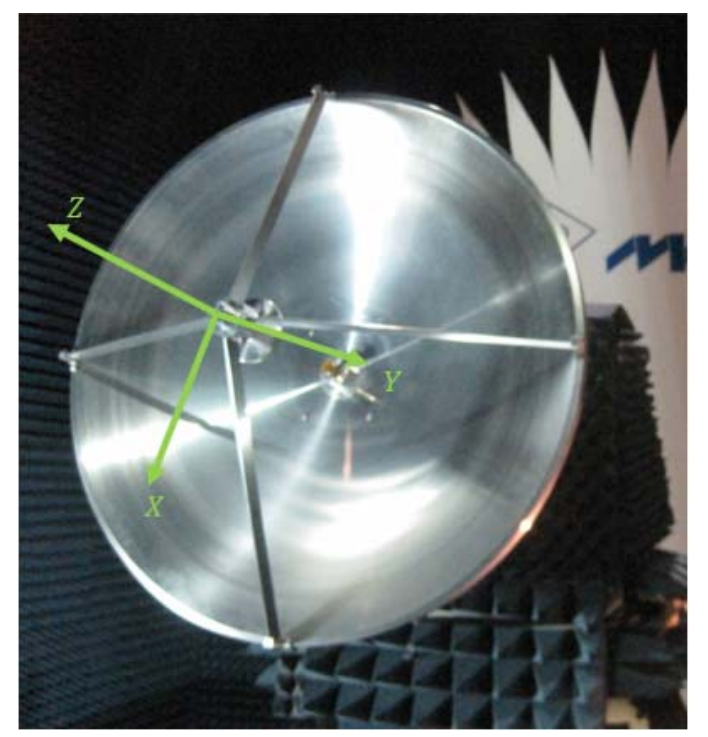

Fig. 6. Antenna used for the algorithm verification and coordiante system definition.
After the evaluation of the CG the coefficients of all SWEs are obtained and then can be evaluated at far-field to obtain the antenna radiation pattern. A $\varphi=0^{\circ}$ cut of this field is shown in Fig. 7 along with the transformed field using a classical spherical near-field to far-field transformation with the information of the complete measurement sphere. The agreement between the two fields is good up values near to $\theta=90^{\circ}$ though there exist small differences due to the truncation of the scan sphere. For $\theta$ values larger than $90^{\circ}$, the proposed algorithm was unable to extrapolate the field, as this corresponds to the truncated solid angle region, so the results have not been depicted.

\section{CONCLUSION}

A near-field to far-field transformation algorithm for arbitrary acquisition surfaces is presented. The algorithm is based on modelling the AUT as a set of unknown multiple spherical wave expansions distributed over the antenna surface. The coefficients of all SWEs are found solving an inverse problem and when found, the field can be evaluated at the infinity to obtain the radiation pattern straightforwardly. It has been shown that with careful choosing of the SWE location and truncation index, the probe effect can be accounted with almost no additional effort without needing to deal with is spherical coefficients and complex rotations. The algorithm has been verified using a real antenna, being able to reconstruct the radiation pattern from the measurement of a half-spherical surface on the near-field.

Using multiple SWEs to model the antenna radiation makes the problem suitable for a multi-level scheme. During the inversion problem, the interaction of near SWEs with the probe could be aggregated in groups following a multilevel structure to speed up the calculations. This is a well-known procedure in the Multi-Level Fast Multipole Method [10] and the same principle can be applied to this problem to reduce the computational complexity of the calculations. This approach is currently under study.

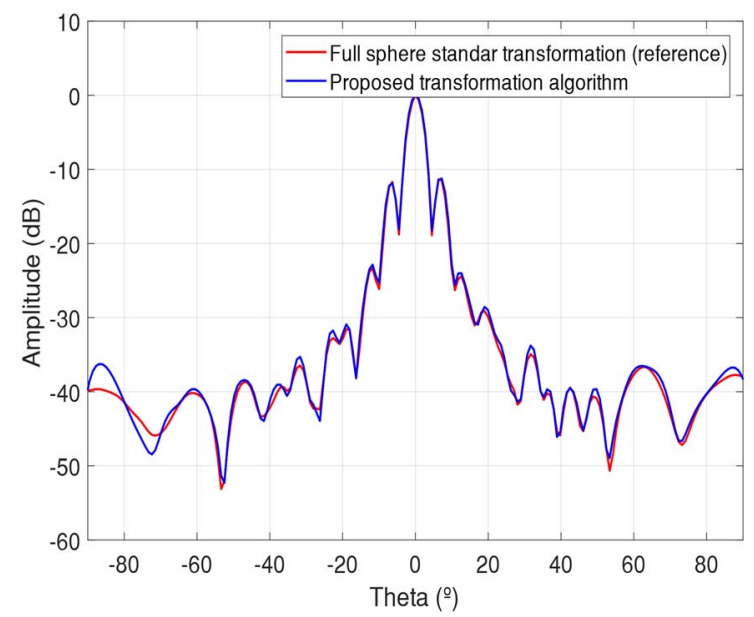

Fig. 7. Comparison of the transformed far-field using the proposed algorithm with a truncated sphere and a classical transformation using the complete shpere. 


\section{ACKNOWLEDGMENT}

Authors would like to acknowledge Universidad Politécnica de Madrid, the Spanish Government, Ministry of Economy, National Program of Research, Development and Innovation for the support of this publication in the projects ENABLING-5G "ENABLING INNOVATIVE RADIO TECHNOLOGIES FOR 5G NETWORKS" (project number TEC2014-55735-C3-1-R) and FUTURE-RADIO "Radio systems and technologies for high capacity terrestrial and satellite communications in an hyperconnected world" (project number TEC2017-85529-C3-1-R) and Madrid Region Government for the project S2013/ICE-3000 (SPADERADAR-CM).

\section{REFERENCES}

[1] O. Breinbjerg, "Spherical near-field antenna measurements-the most accurate antenna measurement technique", IEEE International Symposium on Antennas and Propagation, Puerto Rico, pp. 10191020, June 2016.

[2] J. Hatzis, P. Pelland and G. Hindman, "Implementation of a combination planar and spherical near-field antenna measurement system using an industrial 6-axis robot," AMTA 2016 Proceedings, Austin, TX, USA, 2016, pp. 1-6.

[3] M. G. Fernandez, Y. A. Lopez and F. L. Andres, "On the Use of Unmanned Aerial Vehicles for Antenna and Coverage Diagnostics in Mobile Networks," in IEEE Communications Magazine, vol. 56, no. 7, pp. 72-78, July 2018.

[4] T. K. Sarkar, A. Taaghol, "Near-field to near/far-field transformation for arbitrary near-field geometry utilizing an equivalent electric current and MoM", IEEE Trans. Antennas Propag., vol. 47, no. 3, pp. 566-573, Mar. 1999.

[5] M. Farouq, M. Serhir and D. Picard, "Antenna Far-Field Assessment From Near-Field Measured Over Arbitrary Surfaces," in IEEE Transactions on Antennas and Propagation, vol. 64, no. 12, pp. 51225130, Dec. 2016.

[6] H. Chou, P. H. Pathak, S. Tuan and R. J. Burkholder, "A Novel FarField Transformation via Complex Source Beams for Antenna NearField Measurements on Arbitrary Surfaces," in IEEE Transactions on Antennas and Propagation, vol. 65, no. 12, pp. 7266-7279, Dec. 2017.

[7] J. E. Hansen, Spherical Near-Field Antenna Measurements. London, U.K.: Peter Peregrinus, 1988.

[8] R. Cornelius and D. Heberling, "Correction of non-ideal probe orientations for spherical near-field antenna measurements," 2017 Antenna Measurement Techniques Association Symposium (AMTA), Atlanta, GA, 2017, pp. 1-5.

[9] Y. Saad, Iterative Methods for Sparse Linear Systems, 2nd edition, Society for Industrial and Applied Mathematics, 2003.

[10] J. Song, Cai-Cheng Lu and Weng Cho Chew, "Multilevel fast multipole algorithm for electromagnetic scattering by large complex objects," in IEEE Transactions on Antennas and Propagation, vol. 45, no. 10 , pp. $1488-1493$, Oct 1997. 